\title{
An outbreak of adenovirus type 4 conjunctivitis
}

\author{
ANDREW B. TULLO ${ }^{1}$ AND PETER G. HIGGINS ${ }^{2}$ \\ From the ${ }^{1}$ Bristol Eye Hospital and the ${ }^{2}$ Department of Pathology, Bristol Royal Infirmary
}

SUMMARY An unusually large outbreak of conjunctivitis caused by adenovirus type 4 involved 113 proved cases during a 7-month period. Though the disease was less severe than is commonly seen in epidemic keratoconjunctivitis caused by other serotypes, the cornea was affected in $23 \%$ of cases and symptoms persisted for at least 5 weeks in 6 patients. A concurrent increase in nonocular adenovirus type 4 infections in the Bristol area was not observed. Nine of the cases could have arisen as a result of hospital cross-infection.

Of the 35 distinct serological types of adenovirus only a few are commonly associated with conjunctivitis in temperate regions. Types 3 and 7 are recognised as the principal causes of 'swimming pool' conjunctivitis and pharyngoconjunctival fever. ${ }^{12}$ Types 8 and 19 typically cause the more severe epidemic keratoconjunctivitis. ${ }^{3-5}$ Type 4 and less frequently type 7 give rise to respiratory infections, which may occur in epidemic form, typically among military recruits, being referred to as acute respiratory disease. ${ }^{6}$ Type 4 conjunctival infections are uncommon in the civilian population and have usually been reported as isolated or small numbers of cases during outbreaks caused by other serotypes, ${ }^{7}$ although an outbreak of pharyngoconjunctival fever caused by adenovirus type 4 has occurred in relation to a contaminated swimming pool. ${ }^{8}$

A recent outbreak of adenovirus type 4 conjunctivitis in Bristol involving a large number of persons provided an opportunity to study the range of ocular manifestations resulting from infection with this serotype and to compare it with that previously reported for other adenoviruses.

\section{Materials and methods}

Patients presenting with signs and/or symptoms of possible viral conjunctivitis were seen at the Bristol Eye Hospital Casualty Department. Examination included detailed slit-lamp microscopy and swabs were taken for bacteriological and virological culture.

All swabs for virology were transported in milk saline transport medium and reached the laboratory

Correspondence to Dr A. Tullo, Bristol Eye Hospital, Lower Maudlin Street, Bristol BS1 2LX. within 12 hours. They were examined, as previously described $^{9}$ in cultures of Hep-2 and human embryo diploid fibroblasts, and some were also cultured for TRIC agent.

Serum samples were not taken because the detection of type 4 antibody, especially in single specimens, does not have the same significance as the presence of specific antibody in type 8,10 , or 19 eye infections in temperate climates.

\section{Results}

During the 7-month period from the beginning of March to the end of September 1978, 640 swabs for virus culture were taken from the eyes of patients with possible viral conjunctivitis. These patients all came from the population of approximately 1 million people served by the Bristol Eye Hospital.

Adenovirus type 4 was isolated from eye swabs from 113 of these patients (Fig. 1). The sexes were almost equally affected, with 59 females and 54 males contributing to the total. The average age of the affected patients was 29 years, range 3 months to 67 years. During the same period 22 strains of other adenoviruses were isolated from similar cases. Two belonged to type 1,5 to type 3,1 to type $13 / 30$, and 14 to type 7 , which indicated that a minor concurrent outbreak with the last serotype had occurred.

Of the 113 proved adenovirus cases of type 4 infection swabs had been taken from 10 for isolation of the TRIC agent and from 95 for bacteriology. The results of these investigations were negative except for those from 1 child of 9 months, who yielded a pneumococcus in addition to adenovirus type 4.

In 9 instances infection could possibly have been 


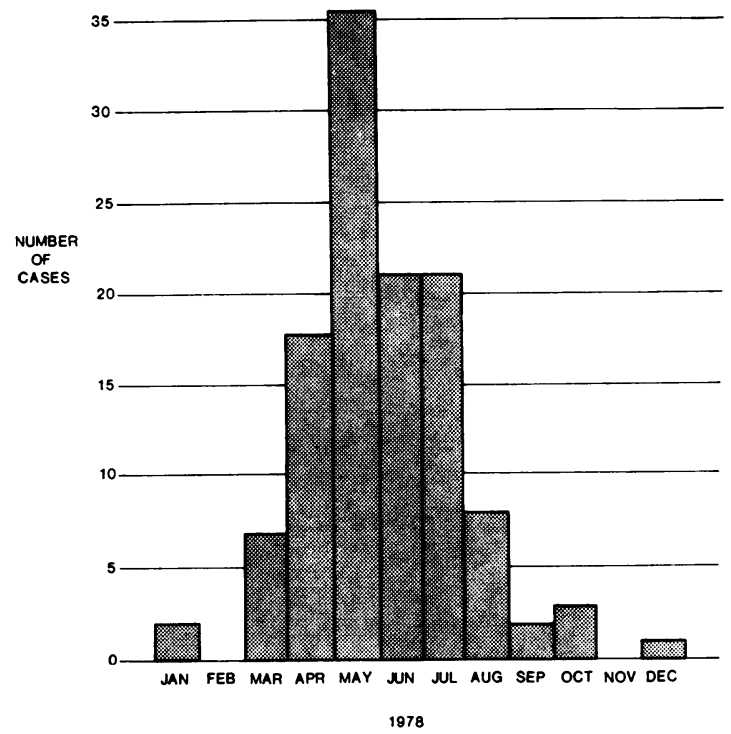

Fig. 1 Distribution of cases during 1978.

acquired in hospital. In these cases patients developed symptoms of conjunctivitis a week or so after attending the outpatients department for some other condition. Five of the patients initially attended with a foreign body in their eye. Three had the foreign body removed by the casualty officer (for further details of 1 see case report below) and 2 by nursing staff. Another 2 patients had irrigation of their tear ducts, also in the treatment room. A further patient, who was responding to treatment for a bacterial periorbital cellulitis, developed a conjunctivitis subsequently shown to be caused by adenovirus type 4 . The remaining case of cross-infection was in a casualty officer. In addition a further 15 patients gave a history of close contact with another person with symptoms of conjunctivitis, 4 of whom were also proved cases attending the Bristol Eye Hospital.

During the period that the 113 isolates of adenovirus type 4 were made from eyes the combined isolations of this serotype by the hospital and the public health laboratories, serving the Bristol area, from sites other than the eye numbered only 3 . The corresponding figures for adenovirus type 7 were 14 strains from the eye and 14 strains from other sites (S. K. R. Clarke, personal communication).

SYMPTOMS (109 INFECTIONS)

Most of the patients presented complaining of soreness and watering of one eye and only a few with a foreign body sensation. The duration of symptoms at the time of presentation ranged from a few hours to 10 days (average 3 days). Symptoms persisted for 14 days on average and ranged from 7 to 42 days. Thirty-four patients progressed to bilateral disease, all within 5 days of onset of symptoms, which in the contralateral eye were typically less severe, though in 8 instances the 2 eyes were equally affected. Fourteen patients $(12 \%)$ had concurrent respiratory symptoms, and 1 was sufficiently ill to be referred to the medical outpatients department (see case report below). Two patients complained of blurring of vision related to corneal opacities.

SIGNS

The frequencies of the various ocular signs are recorded in Table 1. The predominant finding was a follicular conjunctivitis of the lower tarsal conjunctiva. A total of 26 patients had evidence of corneal involvement. Nine of 14 patients with punctate epithelial keratitis did not develop subepithelial lesions; while 12 out of 17 patients who had subepithelial lesions were not observed to have had punctate epithelial keratitis. Corneal subepithelial opacities (Fig. 2) appeared between 3 and 35 days (average 11 days) after the onset of symptoms and had resolved in all but 2 cases after a further 7 to 35 days (average 12 days).

Two patients developed a dendriform epithelial defect early in the course of the infection and went on to develop discrete stromal opacities. Herpes simplex virus was not isolated from either affected eye. One of these patients, a 67-year-old woman, was seen 6 months later, and, although she was asymptomatic, several faint immediately subepithelial opacities were seen in the upper segment of the cornea. This type and distribution of opacities has

Table 1 Distribution and frequency of signs during the course of adenovirus type 4 infections in 109 patients

\begin{tabular}{lll}
\hline Site & Sign & $\begin{array}{l}\text { No. (and \%) of } \\
\text { patients in } \\
\text { whom present }\end{array}$ \\
\hline Lids & Lid swelling & $20(18)$ \\
& Conjunctival hyperaemia & $109(100)$ \\
Conjunctiva & Follicles & $91(83)$ \\
& Papillae & $52(48)$ \\
& Chemosis & $13(12)$ \\
Cornea & Haemorrhage & $9(8)$ \\
& Membrane & $3(3)$ \\
Regional glands & Punctate epithelial keratitis & $14(13)$ \\
\hline
\end{tabular}


been described in the later follow-up of an adenovirus type 10/19 infection. ${ }^{9}$

With the exception of one slowly resolving large plical haemorrhage, all haemorrhages were petechial,

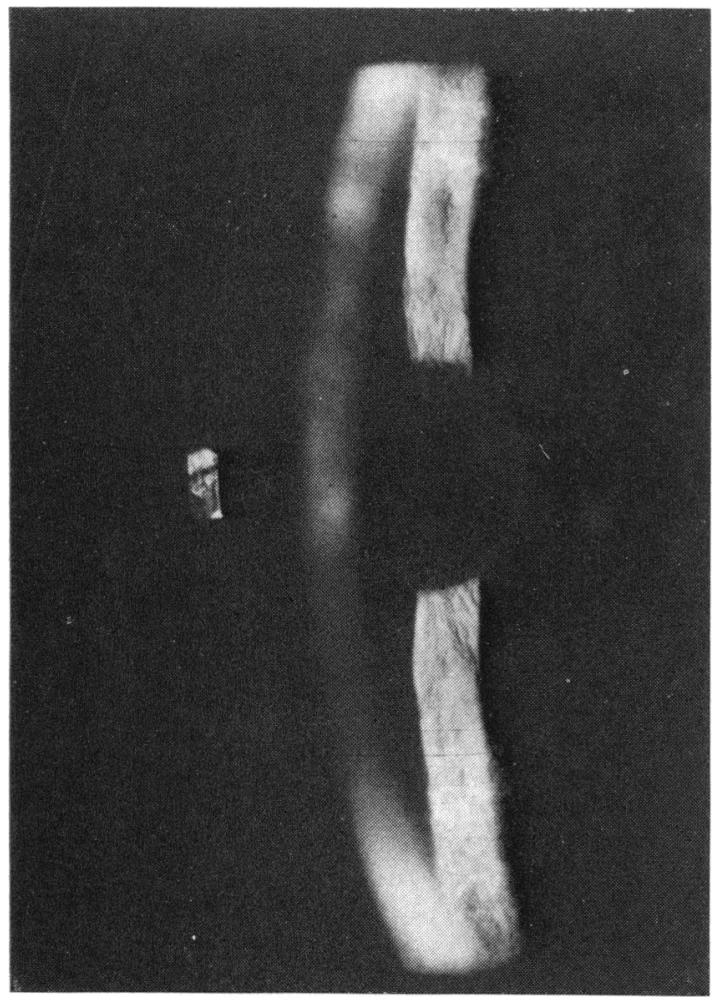

Fig. 2 Slit-lamp photograph showing subepithelial opacities. mainly in the upper tarsal conjunctiva (Fig. 3) and typically lasted for only 2 to 3 days.

\section{CASE REPORT}

A 19-year-old male student presented with a foreign body sensation in the left eye of several hours' duration. An eyelash was removed from under the upper lid. Seven days later he returned giving a 3-day history of his left eye being sore and sticky. Hourly antibiotic drops from his general practitioner had failed to produce any improvement. On examination he was seen to have a marked follicular conjunctivitis with a punctate epithelial keratitis accompanied by a preauricular lymphadenopathy. Eye swabs taken at that time yielded an adenovirus type 4 ; bacterial and chlamydial cultures were negative. On the fourth day of illness he felt feverish and developed a dry cough. He was referred to the medical outpatients department, where a diagnosis of acute bronchitis was made. He was treated with oral tetracycline. Two days later the pain and stickiness of the eye were much less, and examination revealed a chemotic conjunctiva and a clear cornea. Two weeks after onset he felt better and his cough had lessened. On this occasion several subepithelial opacities were noted in his left eye, which were seen to be clearing after a further week, at which time he was asymptomatic.

When seen after 8 months he was well, but examination of the cornea revealed 2 small discrete central subepithelial opacities and 1 similar lesion in the superior peripheral cornea. He was aware of a vague distortion of vision in this eye, though visual acuity was $6 / 4$.

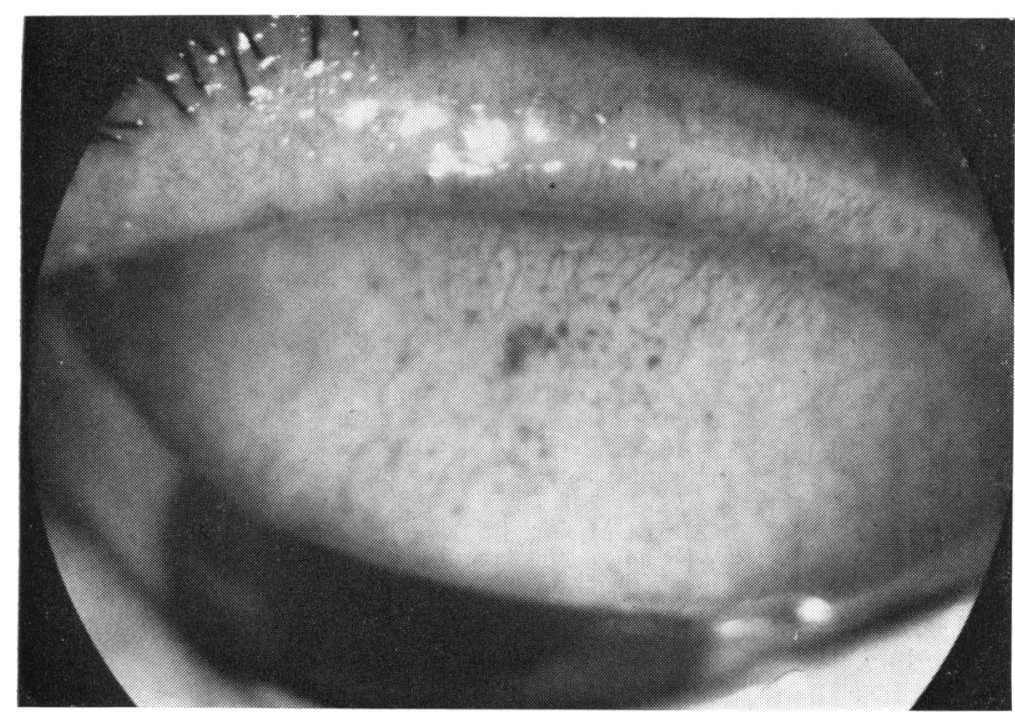

Fig. 3 Petechial haemorrhages in the upper tarsal conjunctiva. 


\section{Discussion}

This outbreak of adenovirus type 4 conjunctivitis was far larger than any previously reported, and the number of strains isolated from eyes, 113, during the 7-month period greatly exceeded the total number of isolates, 34, of this virus from all sites made by the local hospital and public health laboratories over the whole of the preceding 6 years. The apparent low frequency of concurrent adenovirus type 4 infections of the respiratory tract, only 3 isolations from all other sites, could have resulted from biased sampling. However, adenovirus type 7 was isolated with equal frequency from eyes and other sites, 14 strains from each, during the same period. This would indicate that this strain of adenovirus type 4 did have a true predilection for the eye. This situation appears to have been peculiar to the Bristol area in as much as 113 of the 118 isolations of adenovirus type 4 in the Bristol area during a 9-month period were associated with conjunctivitis, but only 67 of the 150 isolates reported to the Communicable Disease Surveillance Centre (CDSC) from the remaining laboratories in England and Wales were from ocular infections. ${ }^{10}$ In summary, $40 \%$ of all adenovirus type 4 infections reported to the CDSC during the first 9 months of 1978 occurred in the Bristol area, as did $63 \%$ of all reported cases of conjunctivitis caused by this serotype.

The 9 instances of probable hospital crossinfection illustrate that it is possible for transmission of the virus to occur by examination alone and that the trauma of a procedure to the eye, for example, removal of a foreign body, increases the likelihood. Unlike the outbreak of adenovirus type 4 PCF related to a swimming pool, ${ }^{8}$ the geographical distribution of cases, excluding the small number of presumed hospital cross-infections, showed no clustering. Furthermore, the typical history was of spontaneous onset, and only $15(13 \%)$ patients gave a history of contact with another person with signs and symptoms of conjunctivitis. This suggests that close contact with an infected eye is not a prerequisite for transmission of the virus. The possibility of asymptomatic ocular carriage acting as a reservoir of adenovirus infection has been raised with type $19 .^{5}$ However, asymptomatic respiratory carriage would provide even greater opportunity for the dissemination of virus and is more likely to occur with adenovirus type 4 , which commonly infects the respiratory tract. However, if asymptomatic carriage was a major factor in the epidemiology of the outbreak it would be necessary to postulate that this particular strain had a greater affinity or pathogenicity for the conjunctiva than previously circulating strains of adenovirus type 4 .

Detailed descriptions of the clinical features of adenovirus type 4 conjunctival infections are scanty, but it has been thought that corneal involvement is rare. Only 1 instance of superficial punctate keratitis occurred in 15 patients with adenovirus type 4 conjunctivitis in Taiwan. ${ }^{7}$ No corneal involvement was observed in a further 20 infections seen during an outbreak of haemorrhagic and follicular conjunctivitis in Rome.11 The observation of petechial haemorrhages in adenovirus conjunctivitis serves as a reminder that acute haemorrhagic conjunctivitis is not pathognomonic of enterovirus infection.

Adenoviruses may be classified according to their haemagglutination properties. ${ }^{12}$ The epidemic types, 8,10 , and 19 , belong to group 2 ; they are transmitted from eye to eye and frequently give rise to corneal opacities which commonly persist for many months. Type 4 adenovirus belongs to another group and behaves somewhat differently. There was no evidence that eye to eye was the common mode of spread of this serotype. The incidence of subepithelial opacities was only $15 \%$, and in all but 2 cases they have resolved within 32 days of their appearance. This is considerably less than the corresponding figures for outbreaks caused by type $8(82 \%)^{3}$ and type $10 / 19(58 \%),{ }^{9}$ which have occurred previously in this region. However, this strain of adenovirus type 4 itself behaved atypically by causing disease in which ocular features predominated.

We are grateful to the staff of the Eye Hospital Casualty Department for collection of swabs and to the staff of the Bristol Royal Infirmary Virus Laboratory for the study of specimens for virus isolation.

\section{References}

${ }^{1}$ Tan Fuzi Y, Mita K, Sata Y, Isikaw Y, Sase Y, Koudo T. Outbreak of acute conjunctivitis caused by adenovirus type 3. Folia Ophthalmol Jpn 1974; 25 : 1-8.

${ }^{2}$ Kendall EJC, Riddle RW, Tuck HA, Rodan KS, Andrews BE, McDonald JC. Pharyngoconjunctival fever; school outbreak in England during the summer of 1955 associated with adenovirus types 3, 7, and 14. Br Med J 1957; ii: $131-6$.

${ }^{3}$ Barnard DL, Dean Hart JC, Marmion VJ, Clarke SKR. Outbreak in Bristol of conjunctivitis caused by adenovirus type 8 and its epidemiology and control. Br Med J 1973; ii: $165-9$.

${ }^{4}$ Editorial: Adenovirus keratoconjunctivitis. Br J Ophthalmol 1977; 61 : 73.

${ }^{5} \mathrm{O}$ 'Day DM, Guyer B, Hierholzer JC, Rossing KJ, Schaffner W. Clinical and laboratory evaluation of epidemic keratoconjunctivitis due to adenovirus types 8 and 19 . Am J Ophthalmol 1976; 81: 27-9.

${ }^{6}$ Hilleman MR, Werner JH. Recovery of a new agent from patients with acute respiratory illness. Proc Soc Exp Biol Med 1954; 85: 183-8. 
${ }^{7}$ Grayston JT, Young Y, Johnson BP, Ko LS. Epidemic keratoconjunctivitis on Taiwan; aetiological and clinical studies. Am J Trop Med 1964; 13: 492-8.

${ }^{8}$ d'Angelo LJ, Hierholzer JC, Keenlyside RA, Anderson LJ, Martone WJ. Pharyngoconjunctival fever caused by adenivorus type 4 ; report of a swimming pool related outbreak with recovery of virus from pool water. $J$ Infect Dis 1979; 140: 42-7.

${ }^{9}$ Tullo AB, Higgins PG. An outbreak of adenovirus kerato- conjunctivitis in Bristol. Br J Ophthalmol 1979; 63: 621-6. ${ }^{10}$ Noah ND. Epidemic infections in 1978. J Infection 1979; 1: 96-102.

${ }^{11}$ Muzzi A, Rocclii G, Lumbroso B, Tosato G, Barbieri F. Acute haemorrhagic conjunctivitis during an epidemic outbreak of adenovirus type 4 infection. Lancet 1975; ii: 822-3.

${ }^{12}$ Rosen $L$. A haemagglutination inhibition technique for typing adenoviruses. Am J Hyg 1960; 71 : 120-8. 